Baltic Astronomy, vol.2, 530-537, 1993.

\title{
A COMPARISON OF THE PREWHITE AND CLEAN PROCEDURES TO FIND THE REAL FREQUENCIES
}

\author{
A. Bruvold \\ Institute of Mathematical and Physical Sciences, University of Tromsø, \\ Auroral Observatory, N-9037 Tromsø, Norway. \\ Received August 1, 1993.
}

\begin{abstract}
A comparison of the prewhite and clean procedures for identifying frequencies in time-series shows that the clean procedure fails if the aliasing effects are bad. The comparison is done using the observations of PG1159-035 of May 1990 and April 1991 which are compared with the WET data of 1989.
\end{abstract}

Key words: methods: data analysis - stars: white dwarfs - stars: individual: PG 1159-035

\section{Introduction}

When we analyze data from WET, or single site data including several nights, we often use the procedure known as prewhite. This procedure implies the calculation of several Fourier Transforms (FT), at least once per frequency identified.

Clean is another procedure for identifying frequencies. This procedure calculates only a few TFs.

With clean, we get a list of frequecies supposed to be present in our data by using two different programs, while prewhite requires use of at least three different programs per frequency identified.

Even though the prewhite programs use less CPU time than the clean programs, prewhite requires longer time in total if the star is multiperiodic, like PG 1159-035. 


\section{Comparison of the programs}

It may seem that there is no sense in using prewhite to identify periods in our data, if clean gives the answer much faster.

To compare these two procedures and to find an answer to this question, I have used both the prewhite and clean procedures on the same sets of data.

The data used were observations of PG 1159-035 in two sets:

\section{Part of the WET run, May 1990 (low priority): \\ 3 different telescopes, \\ 9 runs in 8 days; incomplete.}

\section{Single site, April 1991:}

McDonald Observatory, $30^{\prime \prime}$ and $36^{\prime \prime}$ telescopes,

12 runs in 14 days.

Fig. 1 shows the coverage and window functions for these data sets, in comparison with the coverage and window for PG 1159-035 in the WET run of 1989. It is obvious that the May 1990 window is worse than the single site window for April 1991.

Fig. 2 shows the FTs of the observed data sets. The highest peak in the May 1990 data coresponds to a period of $522 \mathrm{~s}$, not previously observed in PG 1159-035. The highest peak in the April 1991 data corresponds to $538 \mathrm{~s}$, a period also observed in the WET 1989 data (Winget et al. 1991).

I am not going to give a detailed description of the prewhite and clean procedures: it may be found elsewhere (Kepler 1993 and Roberts et al. 1987). I would like just to point out the three most important differences:

1. In prewhite we have to choose one frequency at a time to remove. It does not need to be the highest peak in the FT. To remove the frequency, a sinusoid with the choosen frequency, fitted amplitude and phase is subtracted from the light curve.

2. Clean automaticaly removes the highest peak in the FT, by means of subtracting the window function centered at the frequency with the highest amplitude from the FT.

3. Clean works in the frequency domain, while prewhite works in the time domain.

When comparing the data, I used the same frequency sampling for both procedures. In prewhite, I removed all frequencies identified 

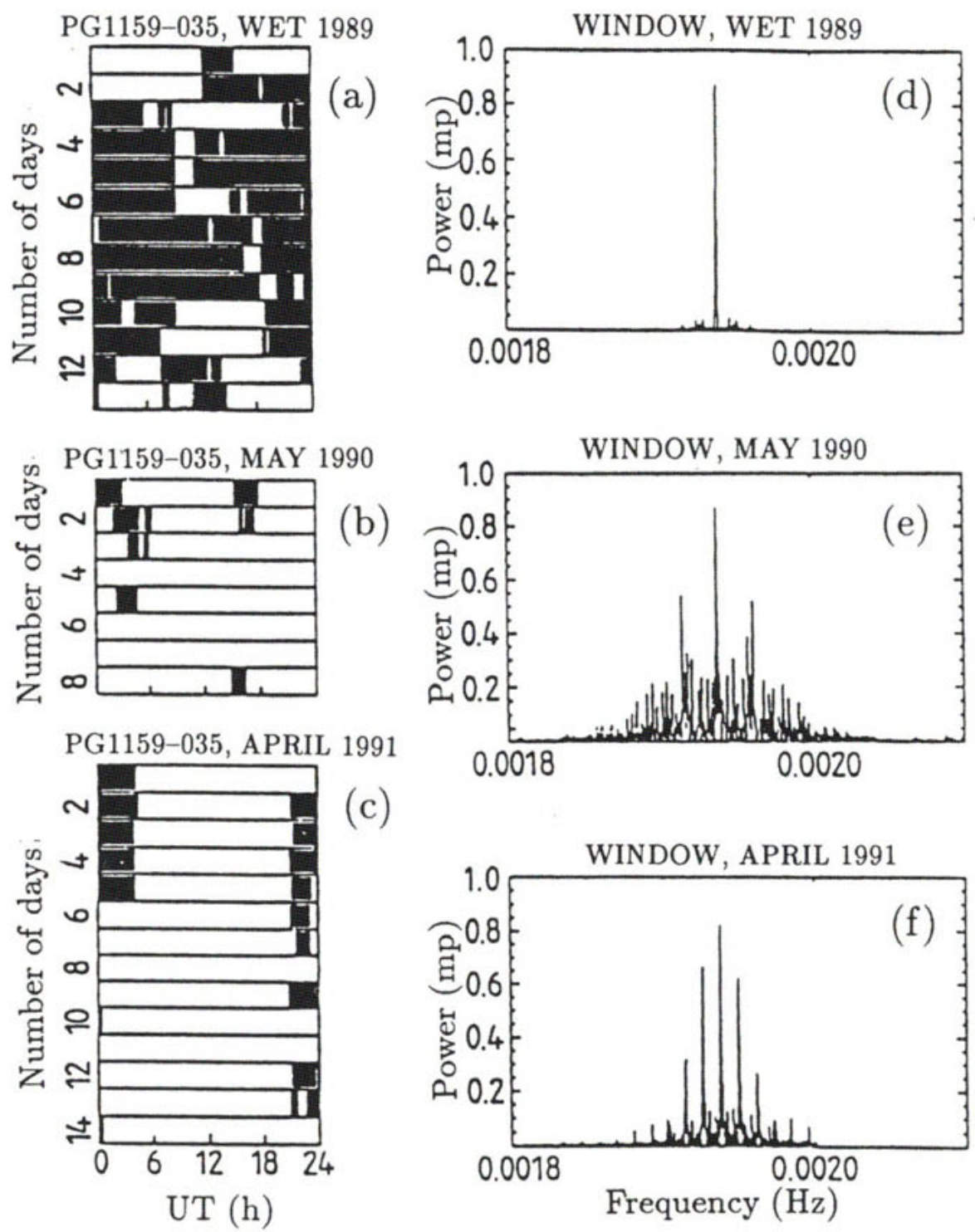

(c)
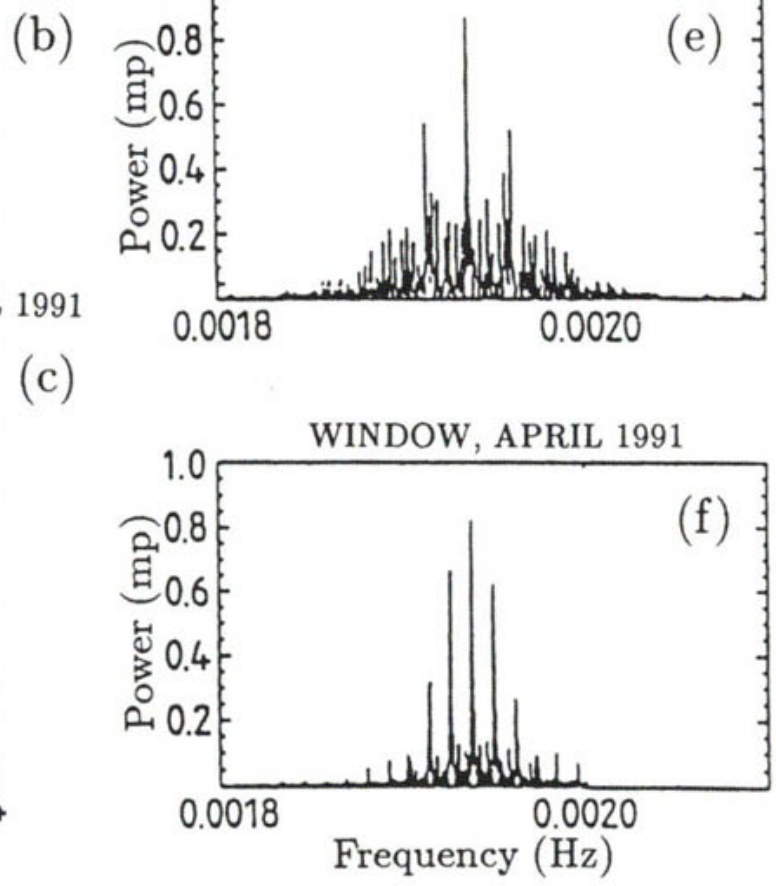

Fig. 1. The coverage and windows for the three sets of observations of PG 1159-035. The coverage is shown on the left-hand side with one panel per day. On the right-hand side there are the window functions for the data sets. Note the difference between the single site data set (f) and the data set of May 1990 (e). 

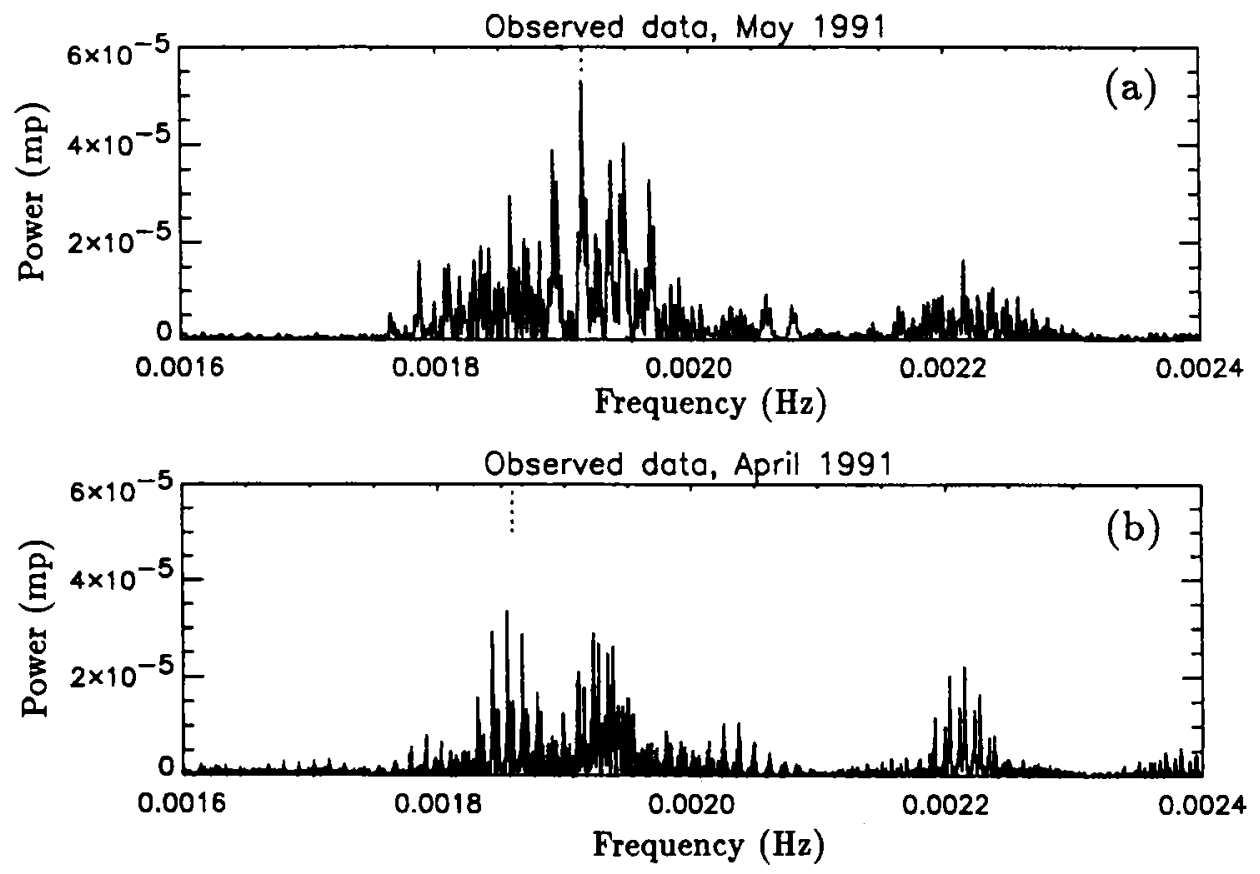

Fig. 2. FT of the May 1990 set (the upper panel) and the April 1991 set (the lower panel). The marked periods are $522 \mathrm{~s}$ in the May 1990 data and $538 \mathrm{~s}$ in the April 1991 data.

in the WET 1989 (Winget et al. 1991), before removing peaks not present in the WET list.

Fig. 3 shows the results of the clean and prewhite procedures for May 1990 (a) and April 1991 (b). The results of clean are multiplied by 4 to give the same power units. Dots show the prewhite results and circles show the clean results. The figures obtained with clean contain only the highest peaks from the frequencies list. We see that the prewhite and clean procedures return completely different frequencies for the May 1990 data (Fig. 3a), while the results for the April 1991 data agree quite well.

In order to check which of the procedures gives the "true" frequencies, I made two sets of synthetic data sampled in the same way as the observed data. First, I processed the single site data (April 1991), using the periods identified by prewhite. The results are shown in Fig. 4. The bars represent the frequencies and amplitudes used in the synthetic data set. The dots are the results from prewhite, the circles are clean results. We can easily see that the frequencies 

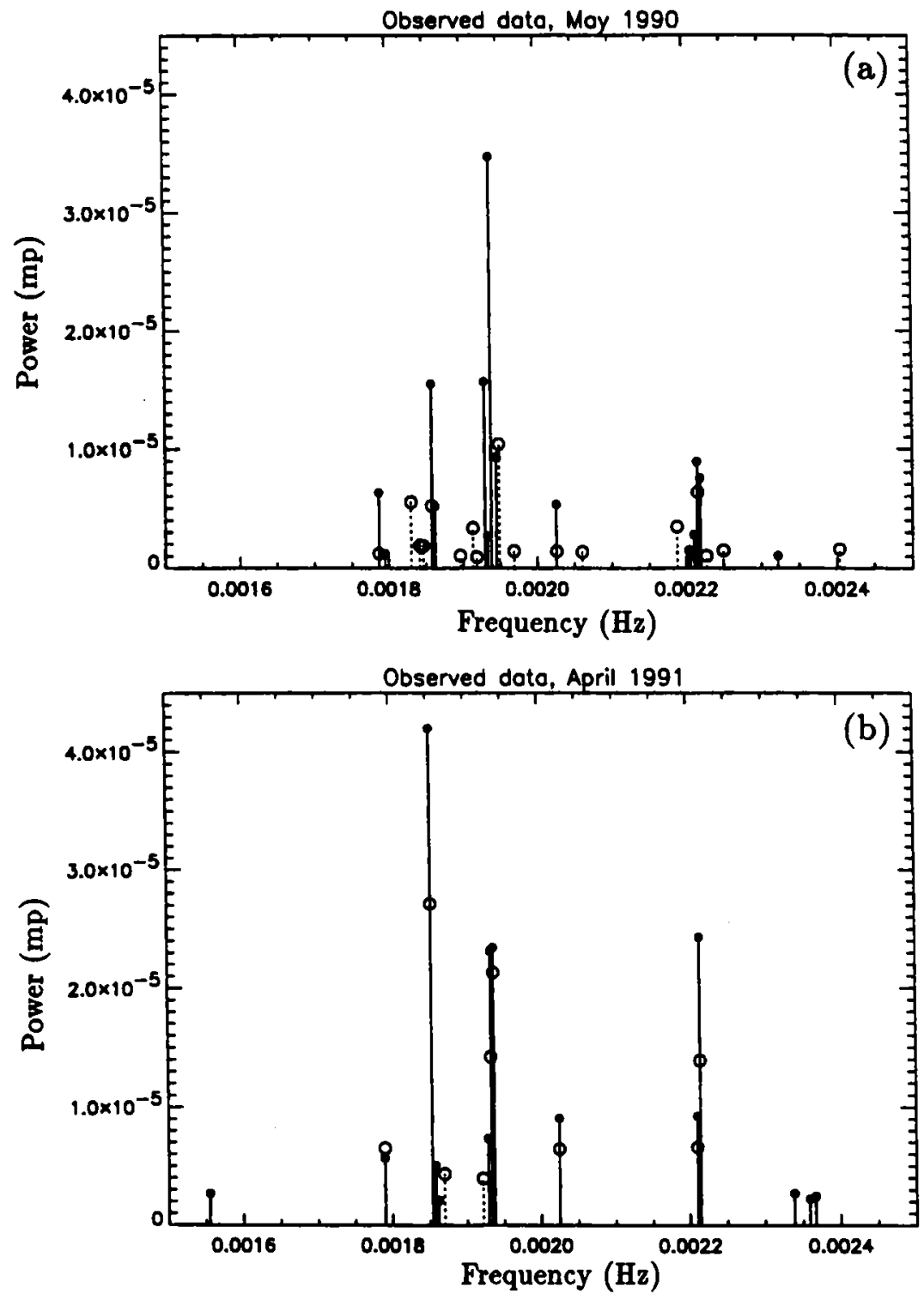

Fig. 3. (a) The results of the clean (circles) and prewhite (dots) procedures used for the May 1990 data; (b) the same for the April 1991 data. 


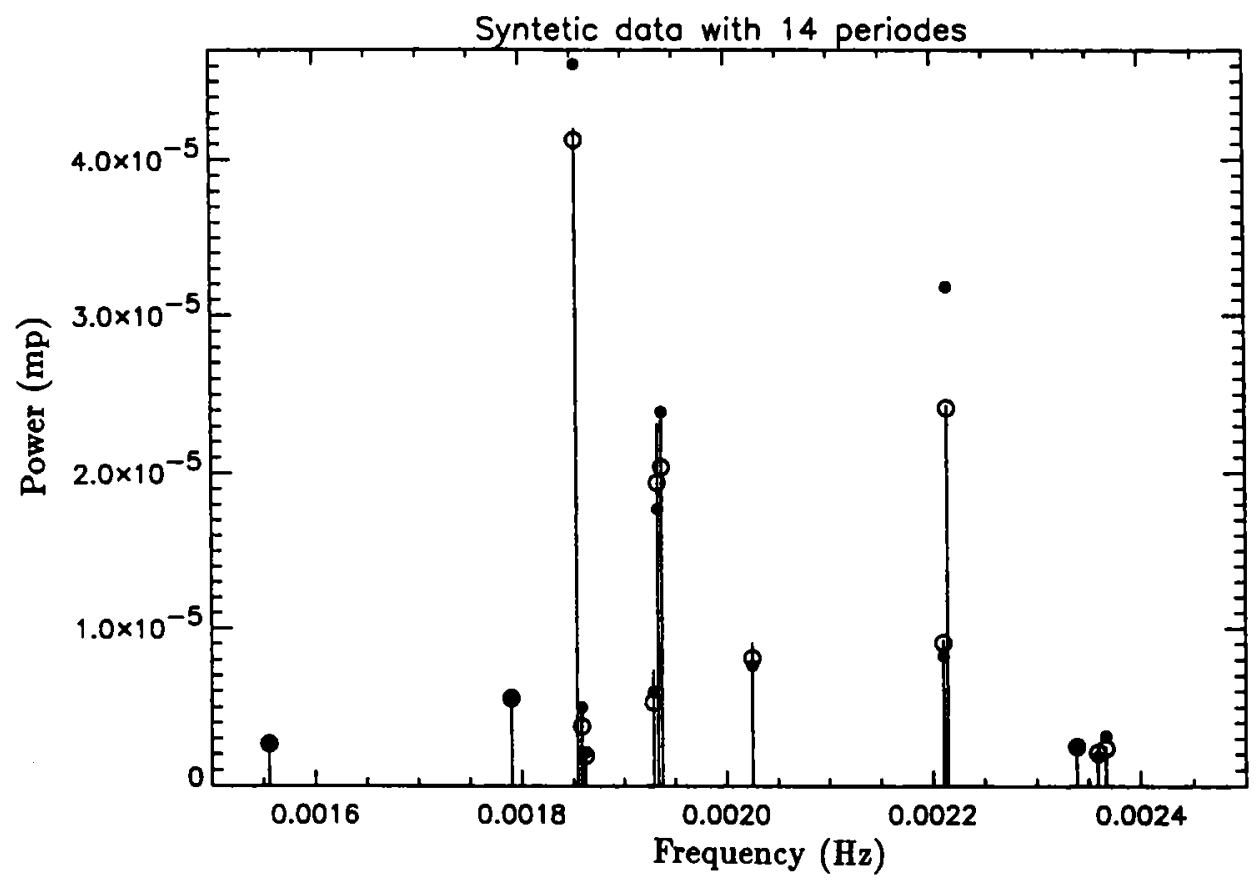

Fig. 4. The results of the prewhite (dots) and clean procedures (circles) on a synthetic data set based on the April 1991 data set (single site). Frequencies and amplitudes in the synthetic file are shown by bars.

identified with both procedures agree. But prewhite gives wrong amplitudes, which could be improved by using a nonlinear fitting to find the phase and amplitude of the frequency.

Then a synthetic data set, sampled in the same way as the May 1990 data, was made, with the frequencies identified by using prewhite on the observed data. The FT of these synthetic data shows a peak at $522 \mathrm{~s}$ (Fig. 5b), although it is not a period used for making the data set. Fig. 5a shows the results when using clean on the synthetic data set. The bars still represent the frequencies and amplitudes in the synthetic data. We find that clean in this case fails to identify the real frequencies and chooses aliasing peaks as real. 

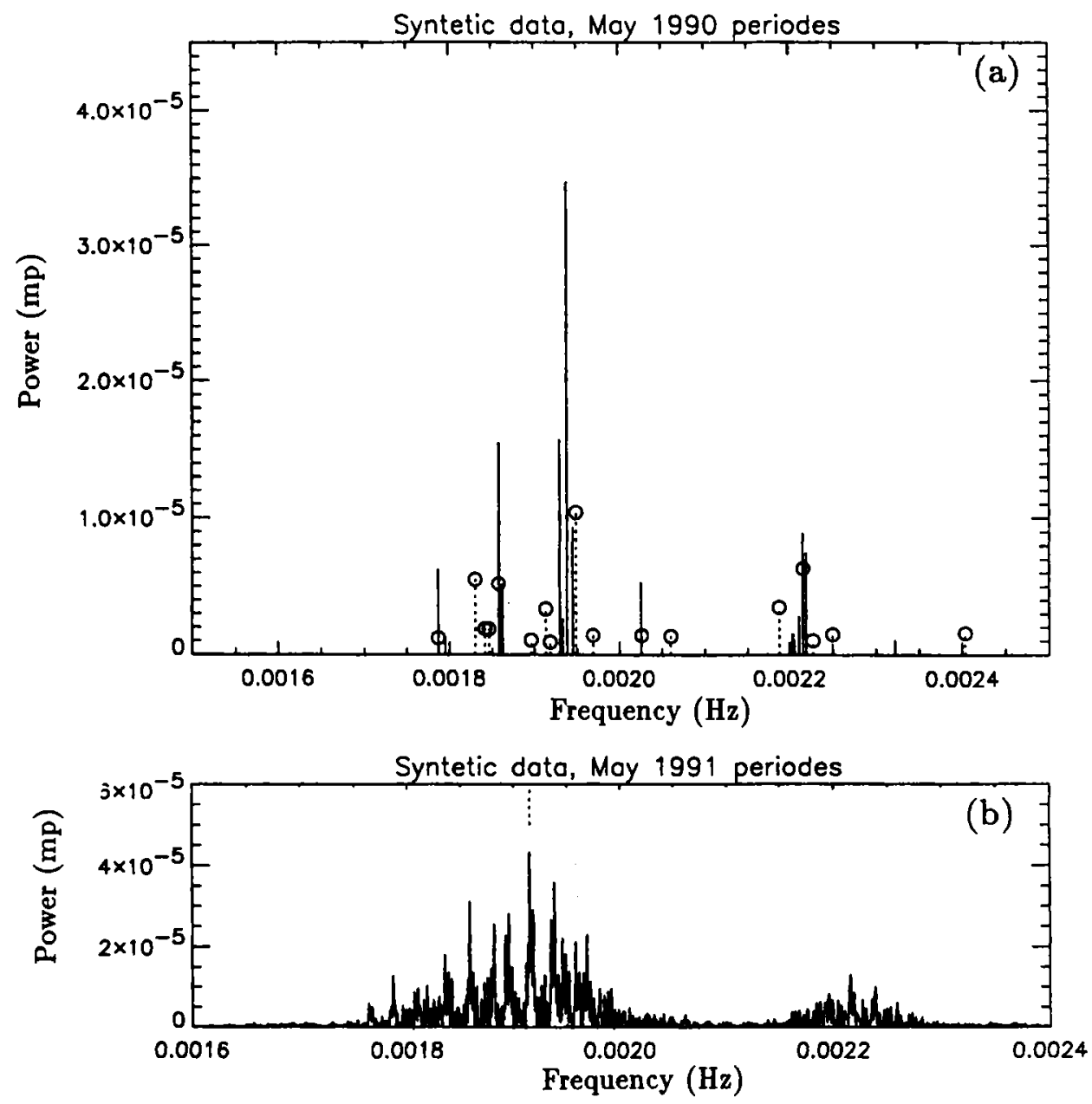

Fig. 5. (a) The clean results (circles) using the synthetic data set based on the May 1990 observations, sampling frequencies and amplitudes in the synthetic file are marked by bars, (b) FT of the synthetic data; the highest peak (an aliasing peak, not existing in the data set) corresponding to $522 \mathrm{~s}$ is marked. 


\section{Conclusions}

As I have shown, the clean procedure gives satisfying results in one case (April 1991), but wrong ones in the other case (May 1990). This procedure cannot be trusted, as it may identify aliasing peaks as the true frequencies.

\section{References}

Bruvold A., Solheim J.-E. 1993, in Precision Photometry, eds. D. Kilkenny, E. Lastovica, J. W. Menzies, South African Astron. Obs. Publ., p. 206. Kepler S. O. 1993, Baltic Astronomy, 2, 515.

Roberts D. H., Lehar J., Dreher J. W. 1987, AJ, 93, 968.

Winget D. E., Nather R. E., Clemens J. C., Provencal J., Kleinman S. J., Bradley P. A., Wood M. A., Claver C. F., Frueh M. L., Grauer A. D., Hine B. P., Hansen C. J., Fontaine G., Achilleos N., Wickramasinghe D. T., Marar T. M. K., Seetha S., Ashoka B. N., O'Donoghoue D., Warner B., Kurtz D. W., Buckley D. A., Brickhill J., Vauclair G., Dolez N., Chevreton M., Barstow M. A., Solheim J.-E., Kanaan A., Kepler S. O., Henry G. W., Kavaler S. D. 1991, ApJ, 378, 326. 